\title{
Design PV system for a small GEO satellite and studying the effect of using different types of propulsion
}

\author{
A. Lotfy, W.Anis, Joseph V. M. Halim \\ Faculty of Engineering, Ain Shams University, Egypt
}

\begin{tabular}{l}
\hline \hline Article Info \\
\hline Article history: \\
Received Nov 29, 2018 \\
Revised Jan 31, 2019 \\
Accepted Feb 19, 2019 \\
\hline
\end{tabular}

Keywords:

Economic

Electric propulsion

GEO satellite

Lithium ion batteries

Multijunction solar cell

\begin{abstract}
This paper presents an optimum design of the solar Photo-Voltaic (PV) power system for small Geostationary Earth Orbit (GEO) satellites using triple junction solar cells and advanced Lithium Ion batteries. The paper applies the proposed system on various propulsion technologies; full chemical, full electrical and hybrid propulsions. This research work studies the capability to fulfil efficiently all the satellite power requirements during both the launching and the on-station phases while reducing the high cost challenge. Since the propulsion type is a key factor for the satellite cost, an economic analysis is demonstrated and investigated in two different strategies. The first scenario fixes the satellite weight and offers the revenue due to the increase in the satellite payload. However, the second scenario evaluates the saving profits due to the reduction in the satellite weight using the same number of satellite transponders. The analytical comparison among the different propulsion techniques shows the superior advantages of using the full electrical satellites.
\end{abstract}

Copyright $(0) 2019$ Institute of Advanced Engineering and Science. All rights reserved.

\section{Corresponding Author:}

A. Lotfy,

Faculty of Engineering,

Ain Shams University,

Nour Mosque 38 Abbasia Next El-Mohamady, Al Waili, Cairo, Egypt.

Email: alotfy718@gmail.com

\section{INTRODUCTION}

Small Geostationary Earth Orbit (GEO) satellites are proposed to accomplish efficiently the geostationary missions with a launch mass of 2 to 3.5 tons and a lifetime of 10 to 15 years. The satellite power system consists of two main parts; the solar array and the storage batteries. The Photovoltaic (PV) solar arrays are the primary source of converting the solar energy to an effective electrical power and the arrays configuration depend on the spacecraft stabilization technique, the type of the orbit and the satellite power requirements [1]. The storage batteries are charged using the generated power of the solar arrays during the daylight and they are used to satisfy all the satellite power requirements during the eclipse durations. In this paper, the proposed solar system uses the triple junction (3J) solar cells, which have highest efficiency and lightest weight. Also, it exploits the benefits of a certain type of the Lithium Ion batteries (Li-ion VES180) due to its low mass and high storage capacity.

Basically, there are three types of propulsion in the satellite market. The first and most common type is the full chemical propulsion (CP), which uses the chemical reactions to produce a flow of fast-moving hot gas. So, the satellite can reach the GEO in few days since the liquid apogee and perigee engines can provide strong pushes using these chemical reactions. Also, the chemical thrusters can perform all the station keeping maneuvers and most of the attitude control operations using this chemical fuel during the satellite lifetime. Therefore, the satellite lifetime is determined by the amount of fuel that can be stored in the propellant tanks. However, all the operations of the satellite subsystems, especially the payload and Telemetry/Command/Ranging (TCR) subsystems, depend on the solar power. The second type is the full 
electrical propulsion (EP), which depends only on the generated power from the solar arrays either during the transfer orbits phase or to perform the station keeping maneuvers, the attitude control corrections and fulfil the subsystems operations. The electric propulsion is limited by the amount of the available power that can be generated by the solar panels and stored in the batteries. This causes that the launching and Geostationary Transfer Orbits (GTO) phase can be extended up to six months to reach the GEO. However, it offers several advantages over the chemical type. First, the economic benefits due to reducing the launching mass, or the opportunity to increase the service payload mass in addition to increasing the operational lifetime [1]. Second, the operational benefits since the electric-propulsion engines and thrusters are more efficient than chemical ones, in the sense that they require much less propellant to produce the same overall effect.

Moreover, the electric thrusters have the ability to regulate the force applied to the spacecraft in very accurate manner and consequently, control the spacecraft's position and orientation along its orbit with an incomparable precision. The third propulsion type is the hybrid, which can be considered as a mix of the previous two types [2]. For this type, the kicks of the transfer orbits are performed using the chemical fuel. However, the station keeping maneuvers, the attitude control operations and the subsystems power requirements depend on the electrical power generated from the solar arrays.

In this paper, an optimum design for the satellite PV system will be applied on GEO satellites and studied for different types of propulsion, including full chemical, hybrid and full electrical. Practically, the design satisfies all the satellite power requirements during the satellite lifetime and its methodology can be summarized in the following steps: First, defining the satellite orbital period and the eclipse duration, used in the presented design; identifying the power demand of the satellite operations, that copes with the satellite lifetime. This includes the power requirements to perform the on-station keeping maneuvers and the attitude control corrections in case of using either hybrid or full electrical propulsions. Also, the required thrust power to perform the GTO kicks, till reaching the GEO, in case of using full electrical propulsion. This is in addition to satisfying the power requirements of the various satellite subsystems; estimating the required area and mass of the satellite solar array, using the selected solar cell type. This is in addition to calculating the corresponding total number of assembled solar cells in the solar array; the last is calculating the optimum size, mass and the required capacity of the selected battery bank.

This paper is organized as follows: Section 2 presents the design of the solar array of the proposed power system and applies it for various types of propulsion. The details of the battery sizing, mass and storage capacity are discussed in section 3 for all the propulsion types. In sections 4 , the economic analysis is presented in two strategies to compare between the propulsion technologies; the first demonstrates the revenue due to the increase in the satellite payload for the same satellite weight and the second shows the saving profits due to the reduction in the satellite weight using the same number of transponders. Finally, the conclusion is drawn in section 5.

\section{THE SOLAR ARRAY DESIGN}

Initially, a full chemical small geostationary satellite is used as a study case. The satellite has a lifetime of 12 years and a total mass of $3028 \mathrm{~kg}$ whereas the fuel mass is $1693 \mathrm{~kg}$. Also, the orbital period is 23.56 hours and the maximum eclipse duration is 72 minutes. The parameters of the proposed power system are demonstrated in the following data:

a. The essential average power is assumed to be $5.859 \mathrm{~kW}$ either during daylight or eclipse, $P_{e}=P_{d}=5.859$ $\mathrm{kW}$, where $P_{e}$ and $P_{d}$ denote the spacecraft power requirements during eclipse and daylight, respectively.

b. A power tracking mechanism is applied by using a Solar Array Drive Mechanism (SADM) to have a peak generated power during daylight.

c. The efficiency of the satellite power subsystem will be roughly $X_{d}=0.8$ and $X_{e}=0.9$ where $X_{d}$ is the efficiency of the spacecraft power subsystem to supply the power from the array to the loads in daylight. However, $X_{e}$ is the efficiency of the spacecraft power subsystem to supply the power from the array to the batteries for charging during daylight and from the batteries to the loads during eclipse [3].

d. The degradation per year for the multi-junction solar cells, like the triple junction (3J) cells, is $0.5 \%$. Also, the nominal value of the Inherent degradation (Id) is 0.77 [4].

The total extent of the generated power, that can be achieved by the solar array during the daylight, is determined by summing the power requirements of the spacecraft during the daylight and the needed power to charge the batteries to accomplish the eclipse power demands. Then, the solar array generated power $P_{s a}$ can be expressed as:

$$
P_{s a}=\frac{\frac{P_{e} T_{e}}{X_{e}}+\frac{P_{d} T_{d}}{X_{d}}}{T_{d}}=7.67 \mathrm{~kW}
$$


where $T_{e}$ and $T_{d}$ are the eclipse and the daylight durations for the satellite in its orbit, respectively. It should be noted that $T_{e}$ equals 72 minutes whereas $T_{d}$ is equal to 1341.6 minutes.

In this paper, the solar array design will be described the following steps:

a. Estimating the output power per unit area of the solar array

The output power per unit area $P_{o}$ can be calculated by multiplying the efficiency of the PV material $(\eta)$ by the solar radiation intensity $\left(G_{s c}\right)$. Since the power efficiency of the $3 \mathrm{~J}$ solar cells is $30 \%$ and $G_{S C}$ equals to $1367 \mathrm{w} / \mathrm{m}^{2}[5], P_{o}$ can be presented as:

$$
P_{o}=\left(G_{s c}\right) \times \eta=410.1 \mathrm{Watt} / m^{2}
$$

b. Calculating the required output power at the beginning of life (BOL) of the satellite

$$
P_{B o l}=P_{o} I_{d} \cos \theta=315.78 \text { Watt }
$$

where $\theta$ is the incidence angle between the vector normal to the solar array and the direction of the sun line. In the presented design, $\theta$ equals zero since the satellite uses a power tracking mechanism.

c. The power degradation factor $\left(L_{d}\right)$ through the satellite lifetime is given as

$$
L_{d}=(1-\text { degradation per year })^{\text {satellite lifetime }}=0.94
$$

d. Estimating the output power at the satellite end of life (EOL)

$$
\text { PEOL }=\text { PBOL Ld }=296.83 \text { Watts }
$$

e. Determining the required area (Asa) and mass (Msa) of the solar array

$$
A_{s a}=\frac{P_{s a}}{P_{E O L}}=25.8 \mathrm{~m}^{2}
$$

The specific performance of the planar array is taken to be $70 \mathrm{~W} / \mathrm{Kg}$ [6]. Consequently, the required mass of the solar array can be evaluated as:

$$
M_{s a}=\left(\frac{1}{70}\right) P_{s a}=109.57 \mathrm{~kg}
$$

f. Calculating the required number (Nsa) of the assembled solar cells in the solar array

The data of the Azur Space for the 3J solar cells is used in the presented design, where the Bus voltage is $V_{B u s}=100 v d c$ and the cell power is $P_{c e l l}=1.24$ Watt. Therefore, the total number of the assembled triple junctions $(3 \mathrm{~J})$ cells in the solar array can be obtained as:

$$
\mathrm{Nsa}=\frac{P_{s a}}{P_{\text {Cell }}}=6185 \mathrm{cells}
$$

In the following discussion, a comparison between the types of propulsion (full chemical, hybrid and full electrical) is performed in power budget, required solar array area and mass points of view.

a. All the previous calculations are performed in case of using full chemical propulsion, where the generated power of the solar arrays is only used to fulfil the power requirements of the satellite subsystems. However, all the maneuvers and attitude control corrections in addition to the transfer orbits kicks are performed using the chemical fuel.

b. Either for full chemical or hybrid satellites, the required amount of the chemical fuel to transfer the satellite during the GTO till reaching the GEO is $1230 \mathrm{~kg}$.

c. For both full electrical and hybrid satellites, the daily North-South and East-West station keeping manoeuvres, in addition to the attitude control corrections every 1-2 days, are performed using the solar arrays power [7]. During the designed satellite lifetime (10-15 years in this paper), the thrusters require about $80-100 \mathrm{mN}$ for the orbital correction operations [8] and about $40-80 \mathrm{mN}$ for the attitude control corrections [1]. To satisfy the previous thrusters force for the orbital and attitude control, the power demand of the satellite will increase about $1.8-2.7 \mathrm{~kW}$ more than the full chemical propulsion type [9].

Int. J. of Adv. in Appl. Sci. Vol. 8, No. 1, March 2019: 54 - 63 
d. Depending on the previous methodology, the satellite using hybrid propulsion needs about $1.8-2.7 \mathrm{~kW}$ extra average power more than using chemical propulsion. This is in order to perform the required station keeping and attitude control corrections during a satellite lifetime of 10-15 years. Using the same formulas of the full chemical satellite, the required generated power $\left(P_{s a}\right)$ from the solar array for the hybrid satellite can be calculated as 10.03-11.21 kW. Consequently, the required area and weight of the solar array will be increased. The calculated results show that the solar array area $\left(A_{s a}\right)$ will increase to 33.8$37.76 \mathrm{~m}^{2}$, the solar array mass $\left(M_{s a}\right)$ will be $143.29-160.14 \mathrm{~kg}$ and the total number of the assembled cells in the solar array (Nsa) will be $8088-9040$ cells.

e. Using full electrical propulsion, the satellite needs approximately $320-540 \mathrm{mN}$ for its GTO kicks till reaching the GEO and about $80-160 \mathrm{mN}$ for the orbit topping [1]. By adding the previously mentioned requirements of the thrusters to satisfy the orbital and attitude control corrections, with a considerable safety margin, the satellite needs about $800 \mathrm{mN}-1 \mathrm{~N}$ during the designed lifetime. This means that there is around 16-20 kW extra average power is required more than the full chemical propulsion [10]. Consequently, the solar array for the electrical satellite should generate power $\left(P_{s a}\right)$ of $28.63-33.87 \mathrm{~kW}$. Therefore, the calculated solar array area $\left(A_{s a}\right)$ is $90.45-114.09 \mathrm{~m}^{2}$, the solar array mass $\left(M_{s a}\right)$ is $312.27-$ $483.86 \mathrm{~kg}$ and the total number of the assembled cells (Nsa) is $23088-27314$ cells.

Figure 1 shows solar array required area versus satellite lifetime using full chemical propulsion.

Figure 2 shows solar array required area versus satellite lifetime using hybrid propulsion. Figure 3 shows solar array required area versus satellite lifetime using full electrical propulsion.

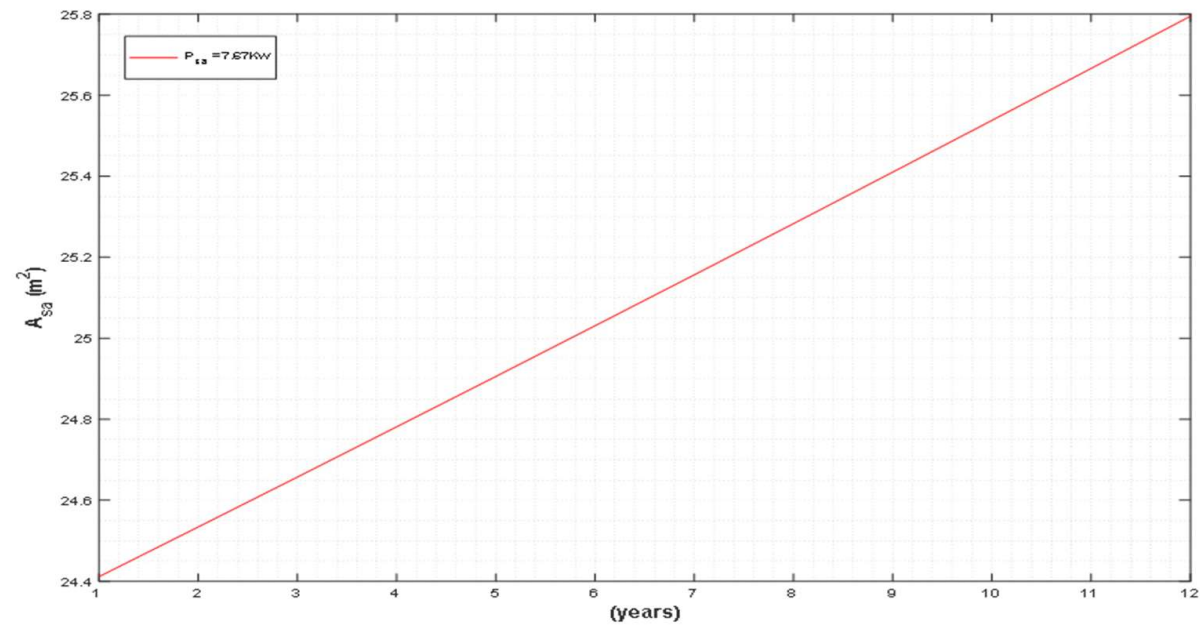

Figure 1. Solar array required area versus satellite lifetime using full chemical propulsion

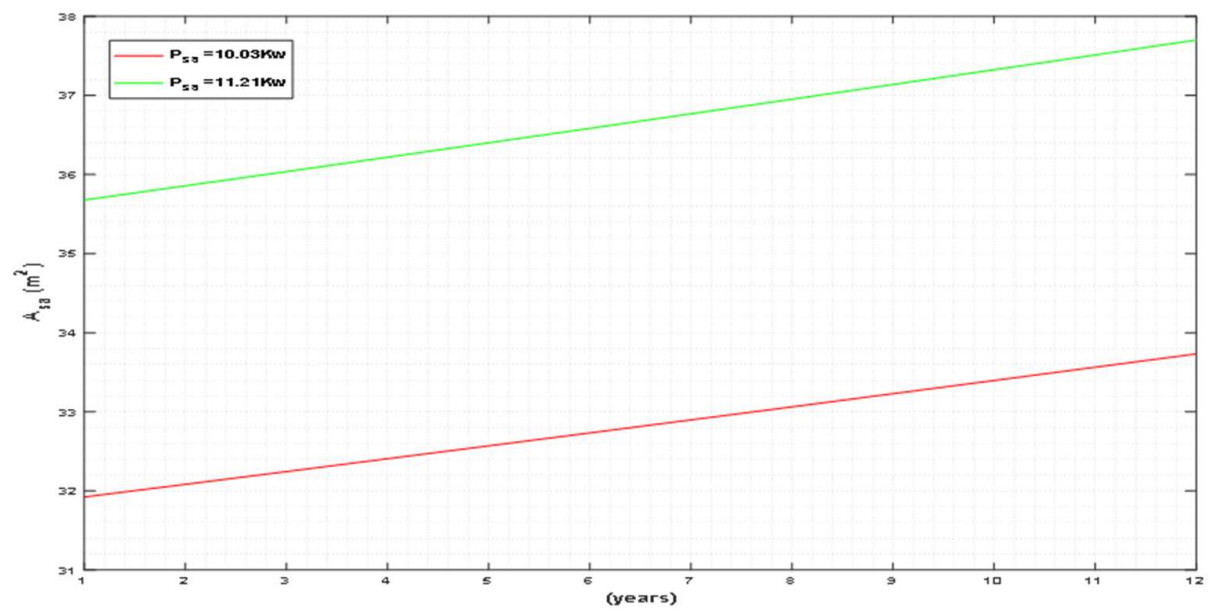

Figure 2. Solar array required area versus satellite lifetime using hybrid propulsion

Design PV system for a small GEO satellite and studying the effect of using ... (A. Lotfy) 


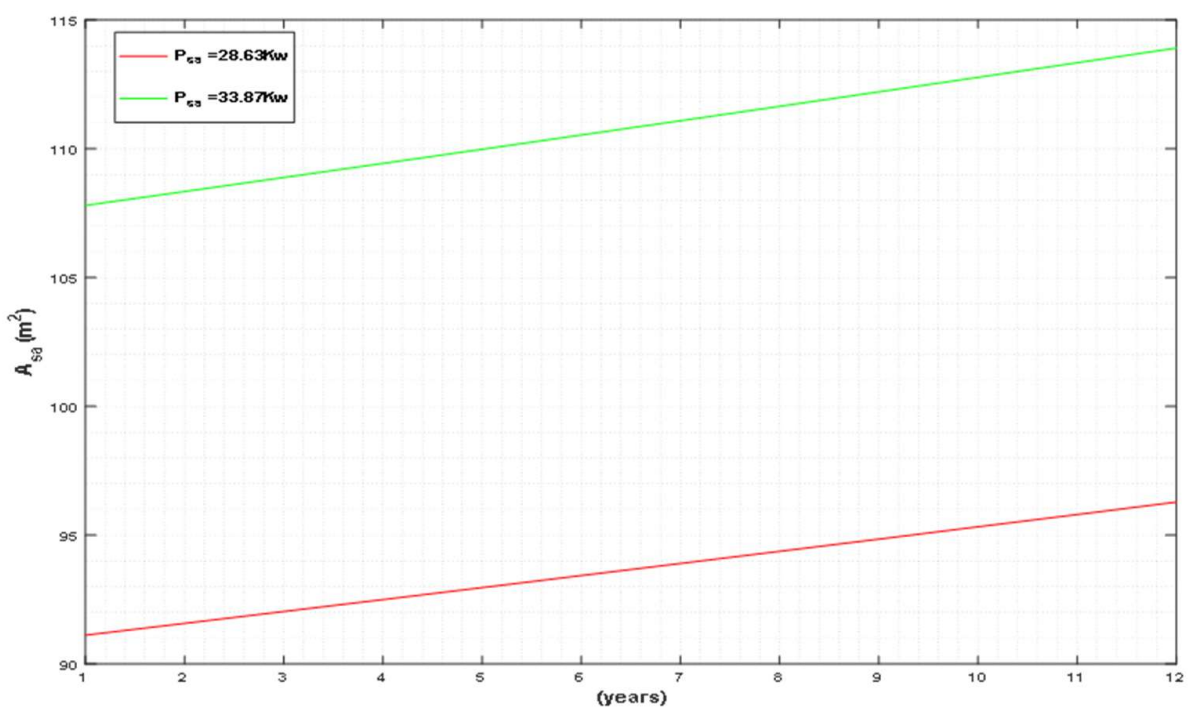

Figure 3. Solar array required area versus satellite lifetime using full electrical propulsion

Figures 1, 2 and 3 illustrate the relation between the required area of the designed solar array versus the satellite lifetime using full chemical, hybrid and full electrical propulsions, respectively. As mentioned before, the solar array in the full chemical satellite generates the power for only the subsystems requirements whereas the chemical fuel is used in all other stages, including transfer orbits, maneuvering and attitude control operations. Also, hybrid satellite uses the solar array to generate power for satellite subsystems, attitude control and maneuvering requirements while the chemical fuel is used only for the transfer orbits. Moreover, the full electrical satellite uses the solar array generated power for all missions, including GTO, maneuvering, attitude control and subsystems power requirements. As a result, the figures show that the largest required area of the solar array will be in case of using full electrical propulsion. Also, the required solar array area for the hybrid satellite is larger than that in case of the full chemical satellite. In addition, there is a linear proportional relation between the designed lifetime of the satellite and the required solar array area since as the satellite lifetime increases, the manufactured solar array area should be increased, respectively.

\section{DESIGN OF THE LI-ION BATTERY}

During the non-shadowing periods, the satellite charges the batteries using the generated power of the solar arrays. The battery storage capacity is used to provide the satellite subsystems with their required power during the eclipse periods. This is in addition to supporting the required station keeping maneuvers and the attitude control operations in the eclipse periods, if needed, in case of using either full electrical or hybrid propulsions.

Initially, the battery sizing for the full chemical satellite can be calculated using the following procedure:

a. Selecting the type of the batteries

Batteries technology can offer many types to the satellite manufacturing, such as Nickel Cadmium (NiCad), Nickel Hydrogen, Lithium Ion (Li-ion), and Sodium Sulfur. Li-ion batteries have the efficient qualification for the space technology and consequently, this type of batteries are currently the common storage devices for various satellite applications. In this paper, an advanced type of Li-ion batteries, called Li-ion VES180, is selected for the presented design due to its low mass and high storage capacity. For the Liion VES180 batteries, the cell voltage is $V_{\text {cell }}=3.6 \mathrm{Vdc}$ and the energy density $E_{d}$ is $150 \mathrm{Wh} / \mathrm{Kg}$ [11]. The Depth of Discharge (DOD) is assumed to be $80 \%$ and the satellite bus voltage is $V_{B u s}=120 \mathrm{Vdc}$ [4].

b. Determining the number of cells (NB) needed in the satellite Li-ion VES180 batteries:

$$
N_{B}=\frac{V_{\text {bus }}}{V_{\text {cell }}}=33.33 \cong 33 \text { cells }
$$


Therefore, the applied satellite bus voltage $V_{B}$ can be expressed as:

$$
V_{B}=N_{B} \times V_{\text {cell }}=118.8 \mathrm{Vdc}
$$

c. The total capacity $(C)$ of the satellite batteries is given by

$$
C=\frac{\left(P_{S a}\right)(\text { max eclipse duration })}{(\text { depth of discharge })\left(V_{B}\right)}=96.84 \mathrm{Ah}
$$

d. The required storage capacity $\left(C_{B}\right)$ of the batteries can be calculated as

$$
C_{B}=C \times V_{B}=11.5 \mathrm{KWh}
$$

e. Estimating the total mass of the satellite batteries (MBatt), required during eclipse

The last step is to estimate the total mass of the batteries, required to satisfy the spacecraft power requirements during eclipse. This can be calculated as shown in the following equation [12].

$$
M_{B a t t}=\frac{C_{B}}{E_{d}}=76.67 \mathrm{Kg}
$$

The previous calculations are executed for the full chemical satellite and in this section, a comparison between the different propulsion techniques will be performed using the same previous steps. As mentioned before, the required generated power $\left(P_{s a}\right)$ from the solar array for the hybrid satellite is 10.03$11.21 \mathrm{~kW}$ whereas for the full electrical satellite, it is $28.63-33.87 \mathrm{~kW}$. Consequently, the calculated results show that the required storage capacity and the mass of the batteries for hybrid propulsion satellite will increase to be 15.04-16.81 kWh and 100.27-112.07 kg, respectively. However, for full electrical satellite, the required capacity and the mass of the batteries are $42.95-50.8 \mathrm{kWh}$ and $286.33-338.67 \mathrm{~kg}$, respectively.

\section{ECONOMIC ANALYSIS}

For a commercial vision, the propulsion is the predominant factor in the spacecraft mass and this will affect the launching cost. In the satellite market, the main goal is to reduce the weight of the satellite while maintaining the possibility of increasing the satellite payload.

\begin{tabular}{cccccc}
\multicolumn{6}{c}{ Table 1. Comparison between the three propulsion types for a GEO satellite of 10-15 years lifetime } \\
$\begin{array}{c}\text { Type of } \\
\text { propulsion }\end{array}$ & Fuel mass $(\mathrm{kg})$ & $\begin{array}{c}\text { Weight of solar } \\
\text { array }(\mathrm{kg})\end{array}$ & $\begin{array}{c}\text { Weight of } \\
\text { Batteries }(\mathrm{kg})\end{array}$ & $\begin{array}{c}\text { Weight of solar } \\
\text { system }(\mathrm{kg})\end{array}$ & $\begin{array}{c}\text { Reduction in } \\
\text { Satellite weight }(\%)\end{array}$ \\
\hline Full chemical & 1693 & 109.57 & 76.67 & 186.24 & - \\
Hybrid & 1230 & $143.29-160.14$ & $100.27-112.07$ & $243.56-272.21$ & $22-24 \%$ \\
Full electrical & - & $312.27-483.86$ & $286.33-338.67$ & $598.6-822.53$ & $50-60 \%$ \\
\hline
\end{tabular}

Table 1. demonstrates a comparison between the propulsion techniques for a GEO satellite of 10-15 years lifetime. As shown, the hybrid satellite offers about $22-24 \%$ reduction in the satellite weight. However, the full electrical satellite can offer about 50-60\% reduction in the satellite weight since it saves the huge mass of the fuel and the propellant tanks in spite of it needs an extra solar system weight, comparing to the full chemical satellite. Therefore, the usage of the full electrical propulsion technology in the satellite market proposes superior economic benefits as follows:

\subsection{Increase the satellite payload for the same satellite weight}

In the economic present value analysis, there are two methods of payment; uniform and geometric gradient payments. This paper will follow the uniform approach, where all the instalments of payment are equal.

$$
X=A *\left(\frac{1-(1+i)^{-Y}}{i}\right)
$$

Denote (X) as the initial payment, (A) is the instalment amount of payment, (i) is the annual interest rate and $(\mathrm{Y})$ is the number of annual instalments, which is considered to be equal to the satellite lifetime [13]. 
The (14) is only effective when the following conditions are fulfilled; the first instalment is paid after one year from the initial payment $(\mathrm{X})$, the amount of the instalments are equal and the durations between the periodic instalments are also equal.

In this paper, the satellite is used for communication purposes. Thus, the satellite payload consists of a number of transponders, each contains a number of channels and each channel offers a revenue every year during the whole satellite lifetime. The following discussion will emphasize the achieved revenue of the full electrical satellite over the full chemical one.

Assume that $w_{S}$ is the satellite saved weight due to using the electrical propulsion with respect to the chemical propulsion and $w_{t}$ is the transponder weight. Thus, $\left(\frac{W_{s}}{W_{t}}\right)$ expresses the excess number of transponders that can be used in the full electrical satellite, for the same total weight of the full chemical satellite. Therefore, the number of excess channels in the full electrical satellite will be $\mathrm{K} \frac{W_{s}}{W_{t}}$, where $(\mathrm{K})$ is the number of channels per transponder.

If the annual renting payment per channel is (R), the annual profit (AP), due to the saved weight, will be expressed as:

$$
\mathrm{AP}=\mathrm{K} \frac{W_{s}}{W_{t}} \mathrm{R}
$$

In case of renting all the channels of the satellite, the extra cost of the full electrical satellite with respect to the full chemical satellite for the designed lifetime is given by:

$$
P_{E C}=P_{1}+P_{2}
$$

where $\left(P_{1}\right)$ is the revenue loss due to the delayed usage of all channels, which is attributable to the GTO duration $(\mathrm{n})$ till reaching the GEO. Also, $\left(P_{2}\right)$ is the extra cost due to excess solar system, which includes the extra solar array and batteries cells.

From (14) and (15), the extra cost of the full electrical satellite for the designed lifetime $\left(P_{E C}\right)$ can be expressed as:

$$
P_{E C}=\mathrm{K} \frac{W_{S}}{W_{t}} \mathrm{R}\left(\frac{1-(1+i)^{-(Y-n)}}{i}\right)
$$

In the previous formula, it should be noted that both of the satellite lifetime (Y) and the GTO duration (n) are expressed in term of years. Also, the revenue loss due to the delayed usage of the satellite channels $\left(P_{1}\right)$ is presented as:

$$
P_{1}=n N_{t} R
$$

where $N_{t}$ is total number of channels in all the satellite transponders. Therefore, the cost of the excess solar systems can be expressed as:

$$
P_{2}=\left[\mathrm{K}\left(\frac{W_{S}}{W_{t}}\right)\left(\frac{1-(1+i)^{-(Y-n)}}{i}\right)\right]-\left(n N_{t} R\right)
$$

Figure 4 represents the analytical results of the economic study for the full electrical satellite with respect to the full chemical one and it is expressed under the following parameters; the satellite has a lifetime of 12 years and 288 channels in all its transponders. Also, the number of satellite transponders is 24 , the GTO duration (n) is six months and the transponder weight $\left(w_{t}\right)$ is assumed to be $12 \mathrm{~kg}$. From table 1 , it is observed that the saved weight $\left(w_{s}\right)$ in the full electrical satellite over the full chemical one in worst case is about $1090 \mathrm{~kg}$.

Int. J. of Adv. in Appl. Sci. Vol. 8, No. 1, March 2019: 54 - 63 


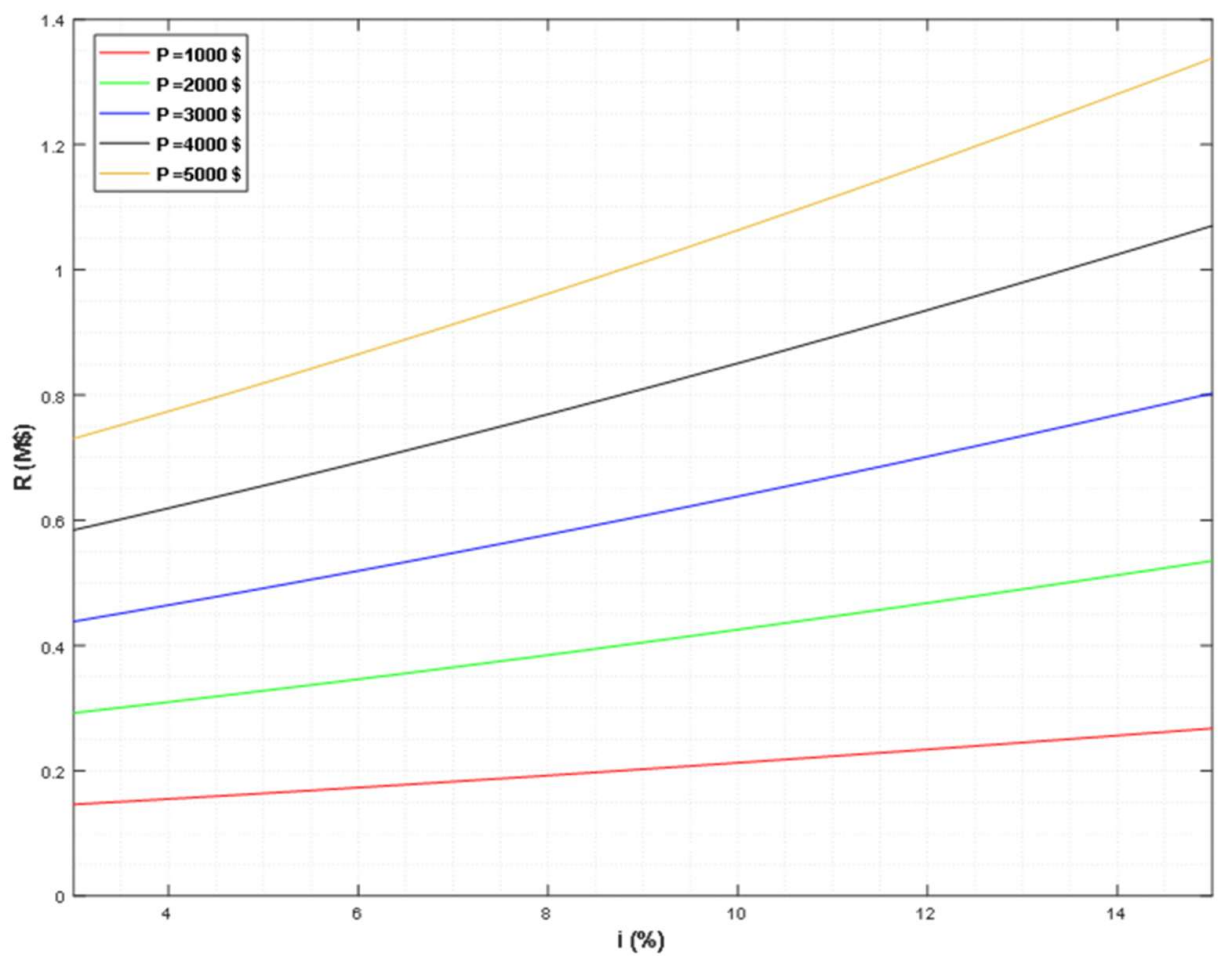

Figure 4. The interest rate versus channel annual renting payment under various values of extra cost due to excess solar system

Figure 4 illustrates the relation between the applied annual interest rate and the annual renting payment per channel for various values of the the extra cost, which is due to excess solar system manufacturing. The results show that as the applied interest rate increases, the estimated amount of the channel renting payment should be increased to have a considerable revenue to the satellite owner. For the same value of the annual interest rate, the figure demonstrates also a linear proportional relation between the extra cost of the solar system and the revenue due to the annual renting payment of the satellite channels. This means that as the extra cost of the solar system increases, which is charged in case of adding more payload, the revenue of the annual channels renting payment increases. This is because the success in reducing the satellite weight gives the opportunity to increase the satellite payload, and consequently add extra channels, through which biggest profits can be achieved from their annual rental.

\subsection{Same number of transponders and satellite weight reduction}

A new economical key factor will be appeared in this case, which is the launching cost. For the same number of satellite transponders, the launching cost will decrease in a descending degree from full chemical to hybrid to full electrical propulsions due to the reduction in the relative satellite weight. As a comparison, the launching cost for a GEO satellite of 24 transponders is 112,705,188 M\$, 96,253,506 M\$ and 75,484,188 M\$ using chemical propulsion, hybrid propulsion and electrical propulsion, respectively. It should be noted that each Kilogram in the GEO satellite launching into space may cost around $€ 30,000 / \mathrm{kg}$ [14]. In this paper, the satellite manufacturing cost is fixed to an estimated value of 250 million dollars, regardless the propulsion type.

The total cost of the satellite, including the launching and the manufacturing costs can be expressed as:

$$
P_{T}=N_{t} \mathrm{R}\left(\frac{1-(1+i)^{-Y}}{i}\right)
$$

where $\left(N_{t}\right)$ is the total number of channels in all the satellite transponders, $(\mathrm{R})$ is the channel annual rent, $(\mathrm{Y})$ is the satellite lifetime and (i) is the annual interest rate. 
Figure 5 illustrates the relation between the annual interest rate versus the channel annual renting payment for the different types of propulsion. It should be noted that PTC, PTH and PTE represent the total cost of the full chemical satellite, the hybrid satellite and the full electrical satellite, respectively. For the same annual interest rate, the lowest annual renting payment per channel is offered in case of the full electrical satellite. The hybrid satellite offers a higher annual channel renting, which reaches its highest payment in case of the full chemical satellite. Therefore, the full electrical satellite presents superior economic advantages due to its competitive annual renting payment of the channels. Consequently, it can easily attract the service providers to rent its channels, comparing to the hybrid and the full chemical satellites of the same coverage areas. This means that the full electrical satellite can achieve a higher occupancy rate and accordingly greater revenues.

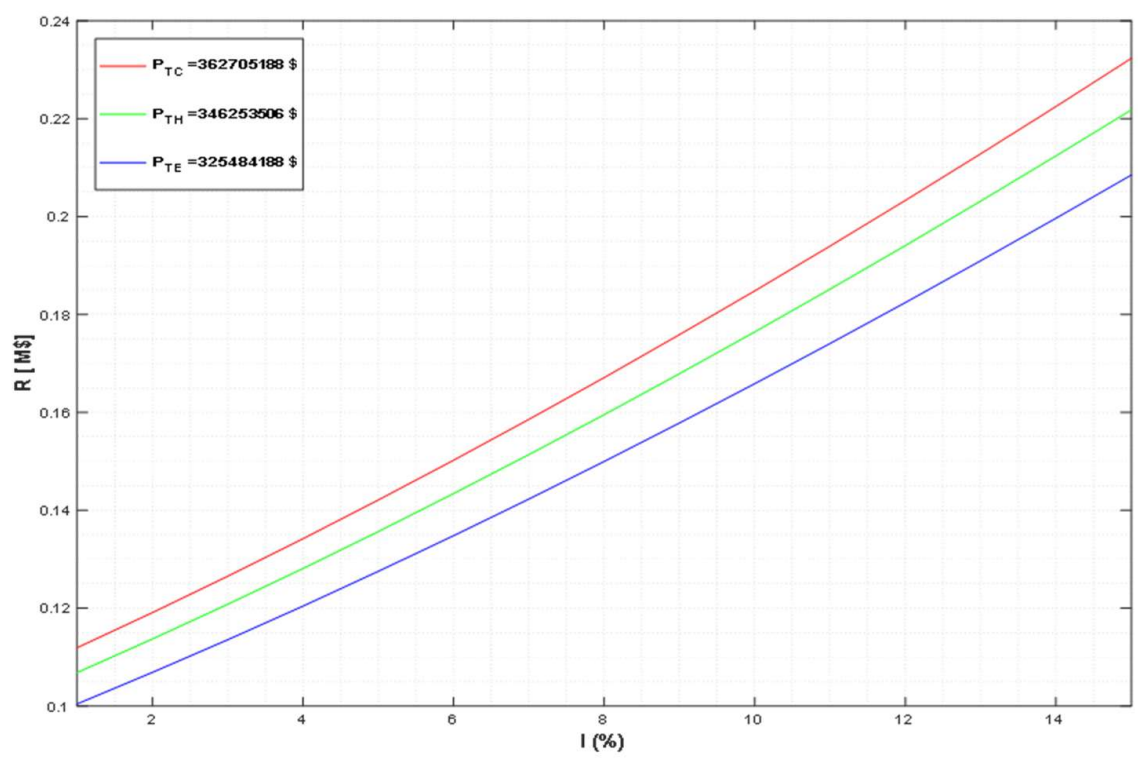

Figure 5. The interest rate versus the channel annual renting payment under various values of the total satellite cost due to using different propulsion techniques

\section{CONCLUSION}

The Electric propulsion is currently one of the most superior technologies and key players in the market of the telecommunication GEO satellites. This paper studied an optimum design of the solar PhotoVoltaic (PV) power system for different types of propulsions; full chemical, hybrid and full electrical satellites. The presented design uses the triple junction (3J) solar cells along with an advanced type of Li-ion battery storage system, Li-ion VES180 batteries. Analytical calculations are performed to evaluate the designed system using the various propulsion types. The technical calculations include the solar array area and mass, the required number of the assembled solar cells in addition to the size and mass of the batteries. Using the full electrical propulsion, the design can efficiently fulfil all the satellite power requirements, during both the transfer orbits and the on-station phases, while offering a big fund in the satellite total cost. This is because in spite of its requirement to have an extra solar array area and batteries capacity, to fulfill the orbit transition and maneuvering operations, it preserves an enormous fuel saving and thus magnificent overall weight reduction that reached $60 \%$, compared to the chemical satellites.

Finally, an economic analysis is applied to compare between the propulsion technologies and it achieves to conclude the benefits and the commercial feasibility in two various scenarios; the first is done by analyzing the economic benefits in case of fixing the satellite weight while increasing the number of transponders. The results draw a high revenue in case of using the electrical propulsion due to the massive increase in the extra number of satellite channels and consequently, in the financial rental returns. However, the second scenario discusses the saving profits due to reducing the satellite weight while using the same number of satellite transponders. The electrical satellite achieves the best results due to the huge fuel saving and consequently, the big fund offered in the launching cost. As a conclusion, the electrical propulsion draws great advantages and profits to the satellite market.

Int. J. of Adv. in Appl. Sci. Vol. 8, No. 1, March 2019: 54 - 63 


\section{REFERENCES}

[1] Hendrick Lubbers, Alexander Schneider, Markus Peukert., "Electric propulsion applications in the smallGEO product line," Deutscher Luft- und Raumfahrtkongress, 2014.

[2] Sheth, Vatsal., "Spacecraft Electric Propulsion-A review," International Journal of Research in Aeronautical and Mechanical Engineering vol.2(9), pp. 43-55, 2014

[3] Mohamed, Ahmed Mokhtar, et al., "Trade-off analysis of low earth orbit spacecraft power supply system by Genetic algorithm." Aerospace Conference, 2016 IEEE. IEEE, 2016.

[4] J. R. Wertz, D. F. Everett and J. J. Puschell, "Space Mission Engineering: The New SMAD," Eds. Hawthorne, CA, USA: Microcosm Press, 2011.

[5] Azur-space "30\% Triple Junction GaAs Solar Cell”, www.azurspace.com.

[6] Donald Rapp, Tom Hamilton, "Solar Cell and Array Technology for Future Space Science Missions". California institute of technology Pasadena, California, pp. 8, 2002.

[7] De Tata, Marco, et al. "SGEO electric propulsion subsystem development status and future opportunities," Proc. 33rd Int. Electr. Propuls. Conf. 2013.

[8] Dudeck, M., et al., "Plasma propulsion for geostationary satellites and interplanetary spacecraft." Romanian Journal of Physics, vol. 56, pp. 3-14, 2011.

[9] Busek space propulsion and systems "BHT-1500 Busek Hall Effect Thruster".

[10] Leporini, A., et al. "Development of a $20 \mathrm{~kW}$ Class Hall Effect Thruster," Proceedings of the Space Propulsion 2016.

[11] Saft-space "Saft batteries... powering outer space for 50 years: extreme performance batteries meeting the demands of space applications", www.saftbatteries.com

[12] Oraby, Osama A., et al., "UASat Solar Array Design and Performance Characteristics". 2014.

[13] L.Blank and A. Tarquin "Basic of Engineering Economics" McGraw Hill, New York, pp.29, 2008.

[14] A. Bacon, "Micro Launch: The Electric Rocket" 13th Reinventing Space Conference 9-13th 2015. 